\title{
Clinical characteristics of the patients with bacteremia due to Moraxella catarrhalis in children: a case-control study
}

\author{
Takanori Funaki ${ }^{1 *}$, Eisuke Inoue ${ }^{2}$ and Isao Miyairi ${ }^{1}$
}

\begin{abstract}
Background: Invasive diseases due to Moraxella catarrhalis are rare in children, even in immunocompromised hosts. Therefore, data regarding clinical characteristics and risk factors of such patients are limited. The aim of this study is to compare the clinical characteristics of patients with bacteremia due to Moraxella catarrhalis against those with bacteremia due to Streptococcus pneumoniae and Haemophilus influenzae.

Methods: We performed a retrospective case-control study to compare patients younger than 18 years of age with positive blood cultures for the three pathogens between June 2008 and May 2014 at our institution. Data regarding patients' demographics and clinical course were collected from their medical records. Three group comparisons, with $M$. catarrhalis as reference, were made by the Fisher's exact test and Wilcoxon rank sum test for discrete and continuous variables, respectively.

Results: There were eight cases of M. catarrhalis, 110 cases of S. pneumoniae (105 patients) and 22 cases of H. influenzae. The $M$. catarrhalis group consisted of six females (75 \%) with a mean age of 16 months. The majority of patients $(7 / 8,88 \%)$ had underlying diseases; however, only one was immunocompromised. Characteristically, six patients (75\%) had medical devices including trans-nasal devices (5/8, $63 \%)$. Univariate analysis revealed that underlying conditions $(P=0.005)$, trans-nasal devices $(P<0.001)$, and lower body weight $(P=0.016)$ and low white blood cell count $(P=0.011)$ at the onset of illness were associated with the $M$. catarrhalis group compared to the S. pneumoniae group. Meanwhile, the higher rates of the patients with underlying conditions and trans-nasal devices were associated with the $M$. catarrhalis group compared to the $H$. influenzae group $(P=0.039, P<0.001$, respectively).
\end{abstract}

Conclusions: The presence of underlying conditions, particularly in those with trans-nasal devices, were characteristic features of patients with bacteremia due to $M$. catarrhalis.

Keywords: Moraxella catarrhalis, Bacteremia, Children, Trans-nasal devices, Underlying disease

\section{Background}

Streptococcus pneumoniae, Haemophilus influenzae and Moraxella catarrhalis are recognized as the three most common bacterial causes of respiratory infection in children. In the pre-vaccine era, S. pneumoniae and $H$. influenzae commonly caused invasive diseases including bacteremia and meningitis. However, since the introduction of the pneumococcal conjugate vaccine (PCV) in

\footnotetext{
* Correspondence: funaki-t@ncchd.go.jp

${ }^{1}$ Division of Infectious Diseases, Department of Medical Subspecialties, National Center for Child Health and Development, 2-10-1 Okura, Setagayaku, Tokyo 1578535, Japan

Full list of author information is available at the end of the article
}

2000 and $H$. influenzae type b (Hib) vaccine in 1987 in the United States, the number of patients with invasive pneumococcal diseases (IPD) and invasive haemophilus disease (IHD) has decreased remarkably [1-5]. Similarly, in Japan, the incidence of patients with IPD or IHD has decreased as a result of the introduction of PCV in 2010 and Hib vaccine in 2008 [6]. However, IPD caused by non-vaccine serotype $S$. pneumoniae and respiratory diseases by non-typable $H$. influenzae have since emerged as a source of concern. Similarly, the relative importance of $M$. catarrhalis as a cause of respiratory tract infection such as otitis media, sinusitis and lower respiratory tract 
disease in otherwise healthy children and elderly persons has been recognized [7]. Invasive diseases due to $M$. catarrhalis are rare in children even in immunocompromised hosts $[7,8]$. Hence, data regarding the clinical characteristics and risk factors of patients with invasive $M$. catarrhalis infection are limited. Identification of patients at risk for invasive infection caused by $M$. catarrhalis, is important when considering empiric therapy because most of the strains are beta-lactamase (penicillinase) producers, in contrast to pneumococcus. The aim of this study is to investigate the characteristics of the patients with bacteremia caused by $M$. catarrhalis compared to those with bacteremia caused by S. pneumoniae or $H$. influenzae.

\section{Methods}

\section{Setting and patients selection}

We retrospectively identified patients with positive blood cultures for M. catarrhalis, S. pneumoniae or H. influen$z a e$ at the National Center for Child Health and Development (NCCHD). The NCCHD is a 490-bed tertiary care children's hospital in Tokyo, Japan, with approximately 12,000 hospitalizations and 35,000 visits to the emergency department each year. The study period was between June 2008 and May 2014. Patients with a disease onset at $\geq 18$ years of age were excluded.

\section{Data collection}

Blood culture results were extracted from the infectious disease surveillance system (Nippon Becton Dickinson Company, Ltd, Tokyo, Japan) at NCCHD. Clinical data of patients who were included were retrospectively collected using electronic medical records, which were reviewed for: 1) patients' demographics including age, gender, body weight, underlying medical conditions and diseases, immunization records for PCV and Hib vaccine, medical devices, presence or absence of immunodeficiency, immunosuppressants, and previous antibiotic use; 2) contact with sick person or people, symptoms, laboratory results at presentation, and focus of infection; and 3) management including treatment and disposition, as well as outcome.

\section{Definitions}

The focus of infection was defined based on the clinical diagnosis or simultaneous identification of the pathogen from the suspected focus (e.g. thoracic empyema, arthritis, and meningitis) and the blood. Occult bacteremia was defined based on the criteria from a previous report. [9] Immunodeficiency was defined as follows: 1) primary immunodeficiency including asplenia; 2) immunosuppressant use such as calcineurin inhibitors, steroids (a dosage equivalent to $\geq 2 \mathrm{mg} / \mathrm{kg} /$ day of prednisone or $\geq 20 \mathrm{mg} /$ day for children who weigh more than $10 \mathrm{~kg}$, for more than 14 days) [10]; and molecular-targeted agents (e.g. rituximab, infliximab); and 3) undergoing or $<6$ months after completion of cancer chemotherapy. Medical devices included any of the following; 1) central venous catheter (peripherally inserted central catheter, short-term (nontunneled) central venous catheter, long-term (tunneled) central venous catheter), 2) urinary catheter, 3 ) intubation (nasotracheal or orotracheal) and tracheostomy, 4) nasogastric tube and elemental diet tube, 5) esophageal thermometer, and 6) other foreign bodies. Onset of disease was defined as community or nosocomial based on 1) whether the patient was hospitalized within 1 month from the onset of illness, and 2) the site at which blood culture sampling occurred (in an outpatient clinic or during hospitalization). Hospital-acquired infection was defined as infection with an onset of illness of more than three days after admission to our hospital. Prior antibiotics use was defined as patients who received antibiotics (oral or intravenous agents) within one week prior to the onset of illness. Duration of fever was defined as the number of days the patient was on antibiotics until defervescence was achieved for more than $24 \mathrm{~h}$ without antipyretics. Recurrence was defined as bacteremia or invasive infection due to the same organism within one month of completion of therapy.

\section{Microbiological methods}

Identification of each organism and antimicrobial susceptibility test were performed at our institution. Blood cultures were inoculated into BacT/ALERT ${ }^{\circ} 3 \mathrm{D}$ bottles (SYSMEX bioMérieux Co., Ltd., Tokyo, Japan). M. catarrhalis, S. pneumoniae and $H$. influenzae were identified using standard methods (BD Phoenix ${ }^{\mathrm{Tx}}$ Automated Microbiology System, Becton, Dickinson and Company, NJ, USA). Antimicrobial susceptibility was determined according to the criteria of the Clinical and Laboratory Standards Institute [11].

\section{Japanese immunization program}

The PCV7 and Hib vaccines became available in Japan in December 2008 on a voluntary basis and became federally funded for children since November 2010. Both vaccines became part of the national immunization program in April 2013. PCV7 was converted to PCV13 in November 2013 [12, 13]. Current immunization schedule recommended by the Japan Pediatric Society is available on the website [14].

\section{Statistical analysis}

Patients' background and disposition data were summarized by mean, standard deviation (SD), interquartile range and proportion as appropriate. The comparisons of $M$. catarrhalis vs. S. pneumoniae and M. catarrhalis vs. $H$. influenzae were made by the Fisher's exact test 
and Wilcoxon rank sum test for discrete and continuous variables, respectively. We considered a $p$-value of less than 0.05 as a meaningful difference. No adjustment for multiplicity was conducted. The $\mathrm{R}$ software (version 3.1.2) [15] was used for statistical calculation.

\section{Ethics}

This study was performed under the institutions opt-out passive consent policy and approved by the ethics committee and the Institutional Board of Privacy and Security at the National Center for Child Health and Development. Patients and guardians wishing to withdraw from the study were able to contact the principal investigator through information provided on notifications publically posted by the institution's ethics committee. Patients were withdrawn based on verbal request from the patient or the guardian (if patients were under 18 years of age at the time of request). Individual participant data is not reported.

\section{Results}

\section{Patients/Cases}

Patients included during the study period were as follows: 1) M. catarrhalis $(n=8), 2)$ S. pneumoniae $(n=$ 105 [110 cases]) and 3) $H$. influenzae $(n=22)$. (Figure 1$)$ Pneumococcal bacteremia gradually decreased after federal funding became available for PCV7 and PCV13.
Bacteremia due to Hib decreased remarkably after introduction of Hib vaccine with no patients observed after April 2012. One patient developed bacteremia due to non-typable $H$. influenzae in May 2014, while bacteremia due to M.catarrhalis occurred sporadically.

\section{Baseline characteristics}

Baseline characteristics among each group are shown in Table 1.

\section{M. catarrhalis}

There were eight cases with bacteremia due to $M$. catarrhalis. Mean age was 16 months and six (75\%) were female. Majority of the patients $(7 / 8,88 \%)$ had underlying diseases. All but one patient were immunocompetent. Characteristically, six patients $(75 \%)$ had medical device, including trans-nasal devices (5/8, $63 \%)$. Trans-nasal devices included nasogastric tube $(n=3)$, elemental diet tube $(n=2)$ and nasotracheal tube $(n=1)$. None of the patients was on antibiotics prior to the onset of illness. Six patients $(75 \%)$ developed hospital acquired infection and two (25\%) were admitted to the pediatric intensive care unit (PICU). Clinical characteristics of the patients with bacteremia caused by $M$. catarrhalis are shown in detail in Table 2. The focus of infection included pneumonia $(n=4)$ and bronchitis $(n=3)$. Two out of eight patients had concomitant infection with other

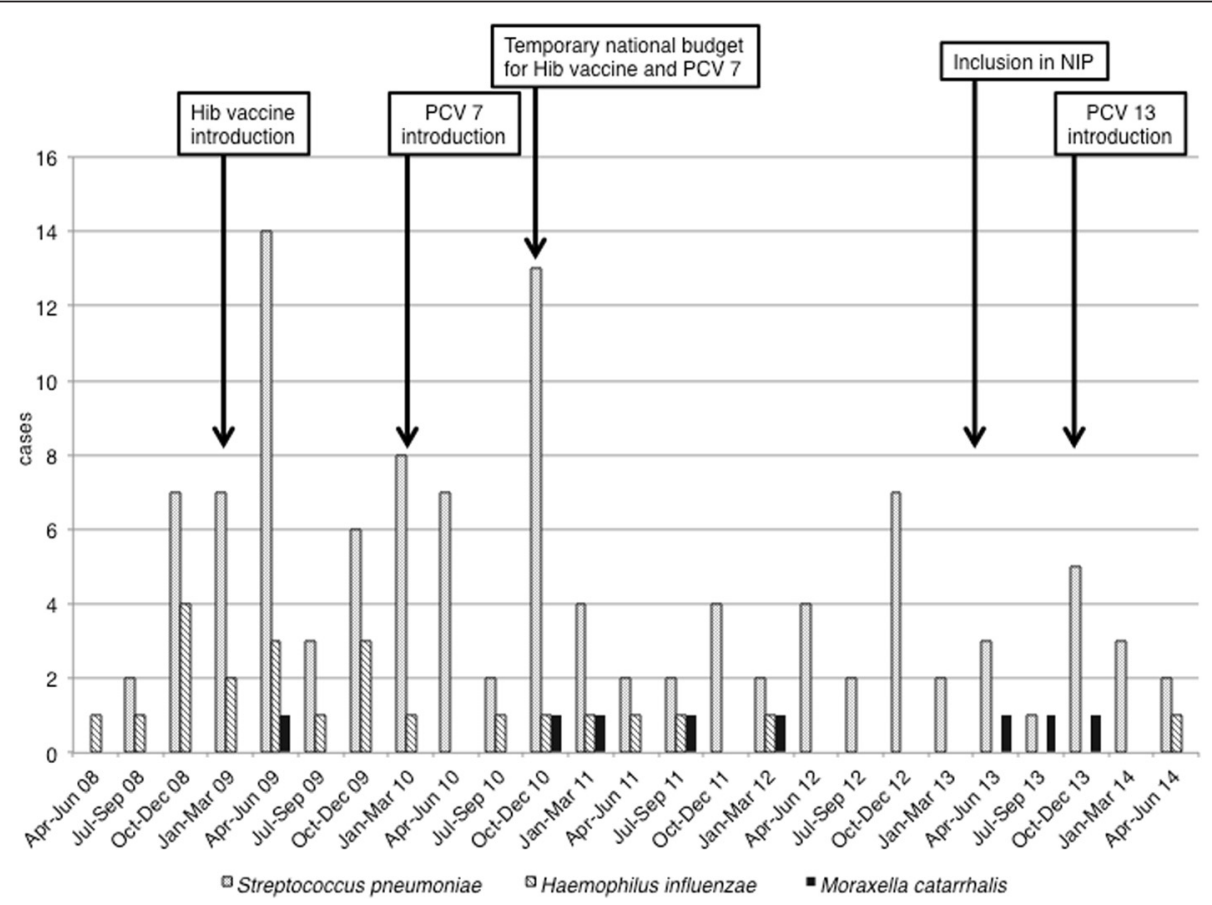

Fig. 1 Quarterly number of patients with bacteremia caused by Moraxella catarrhalis, Streptococcus pneumoniae, or Haemophilus influenzae. X-axis denotes quarterly periods between April 2008 and June 2014. Y-axis denotes the incidence of bacteremia caused by M. catarrhalis, S. pneumoniae and H. influenzae. Abbreviations: Hib, Haemophilus influenzae type b; PCV7, 7-valent pneumococcal conjugate vaccine; PCV13, 13-valent pneumococcal conjugate vaccine; NIP, national immunization program in Japan 
Table 1 Patients' characteristics with bacteremia due to Moraxella catarrhalis, Streptococcus pneumoniae and Haemophilus influenzae

\begin{tabular}{|c|c|c|c|c|c|}
\hline & $\begin{array}{l}\text { M. catarrhalis } \\
(n=8)\end{array}$ & $\begin{array}{l}\text { S. pneumoniae } \\
(n=110)\end{array}$ & $\begin{array}{l}\text { H. influenzae } \\
(n=22)\end{array}$ & $p$-value* & $p$-value \\
\hline Age (months, mean $\pm S D$ ) & $16 \pm 12$ & $35 \pm 32$ & $25 \pm 33$ & - & - \\
\hline Gender (female, \%) & $6(75 \%)$ & $51(46 \%)$ & $6(27 \%)$ & 0.153 & 0.188 \\
\hline \multicolumn{6}{|l|}{ Body weight (kg) } \\
\hline mean $\pm S D$ & $8.31 \pm 2.80$ & $12.49 \pm 6.15$ & $10.20 \pm 3.10$ & $0.016^{\mathrm{a}}$ & 0.188 \\
\hline IQR & $7.45-9.25$ & $8.70-13.60$ & $8.30-10.88$ & & \\
\hline \multicolumn{6}{|l|}{ Vaccination } \\
\hline \multicolumn{6}{|l|}{ PCV7 } \\
\hline YES $(n, \%)$ & $6(75 \%)$ & $25(23 \%)$ & $1(5 \%)$ & $0.004^{\mathrm{a}}$ & $<0.001^{\mathrm{a}}$ \\
\hline \multicolumn{6}{|l|}{ PCV13 } \\
\hline YES $(n, \%)$ & $0(0 \%)$ & $1(1 \%)$ & $0(0 \%)$ & N/A & N/A \\
\hline \multicolumn{6}{|l|}{ Hib vaccine } \\
\hline YES $(n, \%)$ & $5(63 \%)$ & $32(29 \%)$ & $2(9 \%)$ & 0.106 & $0.007^{\mathrm{a}}$ \\
\hline \multicolumn{6}{|l|}{ Underlying diseases } \\
\hline YES (n, \%) & 7 (88 \%) & $39(35 \%)$ & $9(41 \%)$ & $0.005^{\mathrm{a}}$ & $0.039^{\mathrm{a}}$ \\
\hline \multicolumn{6}{|l|}{ Immunodeficiency } \\
\hline YES $(n, \%)$ & $1(13 \%)$ & $14(13 \%)$ & $1(5 \%)$ & 1.000 & 0.469 \\
\hline \multicolumn{6}{|l|}{ Immunosuppressants } \\
\hline YES $(n, \%)$ & $2(25 \%)$ & $7(6 \%)$ & $0(0 \%)$ & 0.114 & 0.064 \\
\hline \multicolumn{6}{|l|}{ Devices } \\
\hline YES $(n, \%)$ & $6(75 \%)$ & $18(16 \%)$ & $0(0 \%)$ & $0.001^{\mathrm{a}}$ & $<0.001^{\mathrm{a}}$ \\
\hline \multicolumn{6}{|l|}{ Trans-nasal devices } \\
\hline YES $(n, \%)$ & $5(63 \%)$ & $7(6 \%)$ & $0(0 \%)$ & $<0.001^{\mathrm{a}}$ & $<0.001^{\mathrm{a}}$ \\
\hline \multicolumn{6}{|c|}{ History of previous intubation } \\
\hline YES $(n, \%)$ & $5(63 \%)$ & $32(29 \%)$ & $4(18 \%)$ & 0.106 & $0.032^{\mathrm{a}}$ \\
\hline \multicolumn{6}{|l|}{ Contact with sick people } \\
\hline YES $(n, \%)$ & $2(25 \%)$ & 45 (41 \%) & $10(45 \%)$ & 0.472 & 0.419 \\
\hline \multicolumn{6}{|l|}{ Pre-existing antibiotics } \\
\hline YES $(n, \%)$ & $0(0 \%)$ & $38(38 \%)$ & $10(45 \%)$ & $0.049^{a}$ & $0.029^{\mathrm{a}}$ \\
\hline \multicolumn{6}{|c|}{ Hospital acquired infection } \\
\hline YES (n, \%) & $6(75 \%)$ & $18(16 \%)$ & $1(5 \%)$ & $0.001^{\mathrm{a}}$ & $<0.001^{\mathrm{a}}$ \\
\hline \multicolumn{6}{|l|}{ Symptoms } \\
\hline Rhinorrhea $(\mathrm{n}, \%)$ & $2(25 \%)$ & $53(48 \%)$ & $3(14 \%)$ & 0.281 & 0.589 \\
\hline Cough (n, \%) & $5(63 \%)$ & $44(40 \%)$ & $5(23 \%)$ & 0.274 & 0.078 \\
\hline Convulsion (n, \%) & $1(13 \%)$ & $31(28 \%)$ & $8(36 \%)$ & 0.445 & 0.374 \\
\hline Vomiting (n, \%) & $2(25 \%)$ & $14(13 \%)$ & $1(5 \%)$ & 0.296 & 0.166 \\
\hline Diarrhea (n, \%) & $1(13 \%)$ & $6(5 \%)$ & $2(9 \%)$ & 0.350 & 1.000 \\
\hline \multicolumn{6}{|l|}{ Body temperature } \\
\hline $\operatorname{Tmax}\left({ }^{\circ} \mathrm{C}\right.$, mean) & 38.9 & 39.3 & 39.0 & 0.176 & 0.557 \\
\hline IQR & $38.6-39.2$ & $38.7-40.0$ & $38.5-39.8$ & & \\
\hline \multicolumn{6}{|l|}{ Focus of infection } \\
\hline Occult bacteremia & $0(0 \%)$ & $40(36 \%)$ & $2(10 \%)$ & 0.050 & 1.000 \\
\hline Pneumonia & $4(50 \%)$ & 19 (17\%) & $5(22 \%)$ & 0.046 & 0.195 \\
\hline Meningitis & $0(0 \%)$ & $4(4 \%)$ & $8(36 \%)$ & 1.000 & 0.071 \\
\hline
\end{tabular}


Table 1 Patients' characteristics with bacteremia due to Moraxella catarrhalis, Streptococcus pneumoniae and Haemophilus influenzae (Continued)

\begin{tabular}{|c|c|c|c|c|c|}
\hline Otitis media/Sinusitis & $0(0 \%)$ & $13(12 \%)$ & 1 (5 \%) & 0.596 & 1.000 \\
\hline Others & $3^{b}(38 \%)$ & $10^{c}(9 \%)$ & $5^{d}(22 \%)$ & 0.048 & 0.643 \\
\hline Unknown & $1(12 \%)$ & $24(22 \%)$ & $1(5 \%)$ & 1.000 & 0.469 \\
\hline \multicolumn{6}{|l|}{ Empiric antibiotic therapy ${ }^{\mathrm{e}}$} \\
\hline Aminopenicillins $^{f}$ & $0(0 \%)$ & $19(17 \%)$ & $0(0 \%)$ & 0.351 & N/A \\
\hline Beta-lactam/Beta-lactamase inhibitor combinations ${ }^{g}$ & $3(38 \%)$ & $8(7 \%)$ & $1(5 \%)$ & 0.026 & 0.048 \\
\hline Second generation cephalosporins ${ }^{\text {h }}$ & $1(12 \%)$ & $2(2 \%)$ & $0(0 \%)$ & 0.191 & 0.267 \\
\hline Third generation cephalosporins ${ }^{i}$ & $3(38 \%)$ & $71(65 \%)$ & $17(77 \%)$ & 0.147 & 0.078 \\
\hline Others ${ }^{j}$ & $1(12 \%)$ & $17(15 \%)$ & $8(36 \%)$ & 1.000 & 0.374 \\
\hline No treatment & $0(0 \%)$ & $3(3 \%)$ & $2(9 \%)$ & 1.000 & 1.000 \\
\hline Initiation with combination therapy & $0(0 \%)$ & $10(9 \%)$ & $6(27 \%)$ & 1.000 & 0.155 \\
\hline Duration of fever after treatment (days, mean $\pm \mathrm{SD}$ ) & $2.63 \pm 0.92$ & $1.86 \pm 1.00$ & $3.77 \pm 3.61$ & $0.012^{\mathrm{a}}$ & 0.599 \\
\hline $\mathrm{IQR}$ & $2-3$ & $1-2$ & $2-4.75$ & & \\
\hline WBC $(/ \mu L$, mean $\pm S D)$ & $14,774 \pm 13,750$ & $19,706 \pm 7862$ & $17,805 \pm 10,173$ & & \\
\hline $\mathrm{IQR}$ & $9395-12,158$ & $14,548-24,318$ & $9468-22,988$ & $0.011^{\mathrm{a}}$ & 0.277 \\
\hline ANC $(/ \mu L$, mean $\pm S D)$ & $11,395 \pm 13,884$ & $14,690 \pm 7569$ & $12,057 \pm 7662$ & & \\
\hline $\mathrm{IQR}$ & $5220-9049$ & $8984-19,408$ & $6964-15,575$ & $0.025^{\mathrm{a}}$ & 0.237 \\
\hline $\mathrm{CRP}(\mathrm{mg} / \mathrm{dL}$, mean $\pm \mathrm{SD})$ & $1.86 \pm 1.47$ & $5.65 \pm 6.68$ & $8.09 \pm 8.60$ & & \\
\hline $\mathrm{IQR}$ & $1.00-2.68$ & $1.00-8.03$ & $1.30-15.08$ & 0.114 & 0.091 \\
\hline \multicolumn{6}{|l|}{ Hospitalization } \\
\hline YES $(n, \%)$ & $7(88 \%)$ & $84(76 \%)$ & $14(64 \%)$ & 0.680 & 0.374 \\
\hline \multicolumn{6}{|l|}{ ICU admission } \\
\hline YES $(n, \%)$ & $2(25 \%)$ & $9(8 \%)$ & $1(5 \%)$ & 0.162 & 0.166 \\
\hline \multicolumn{6}{|c|}{ 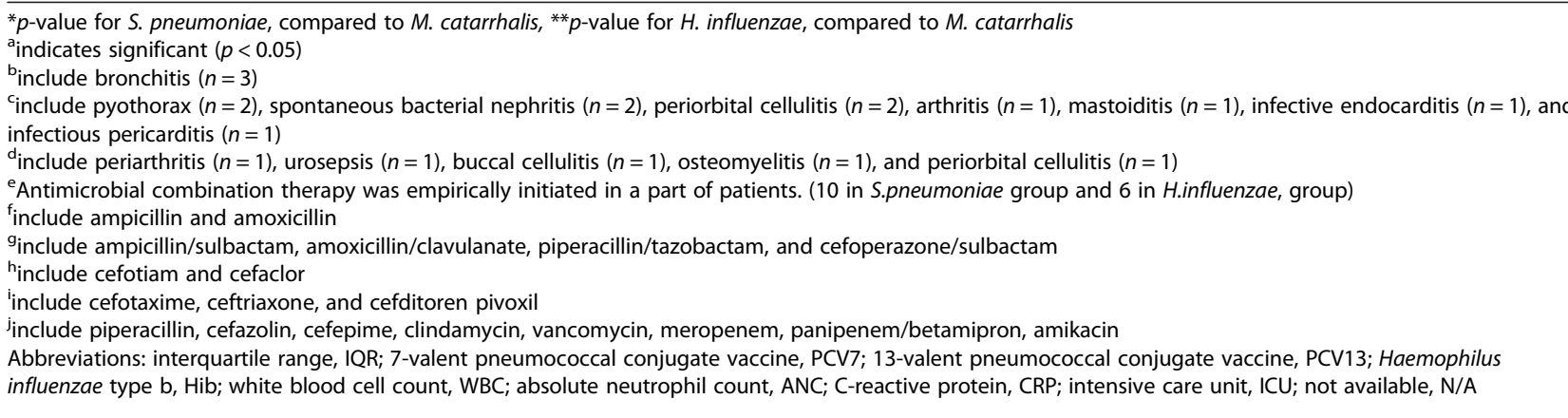 } \\
\hline
\end{tabular}

pathogens; rotavirus gastroenteritis $(n=1)$ and respiratory syncytial virus infection $(n=1)$. All isolated strains of $M$. catarrhalis were beta-lactamase producers. All but one patient were empirically treated with appropriate antibiotics for M. catarrhalis (cefotaxime [ $n=2]$, ampicillin/sulbactam $[n=2]$, ceftriaxone $[n=1]$, piperacillin/ tazobactam $[n=1]$, cefotiam $[n=1]$, and vancomycin $[n$ $=1]$ ). The patient empirically treated with vancomycin (for just one day) was switched to cefotaxime soon after the identification of positive blood culture. Three patients were switched to oral antibiotics. The median duration of treatment was 14 days (range: 13-14). All patients recovered without any recurrence or death after the completion of antimicrobial therapy.

\section{S. pneumoniae}

There were 110 cases (105 patients) with bacteremia due to S. pneumoniae. Mean age was 35 months and 51 (46\%) were female. Of all patients included, only 26 patients $(24.8 \%)$ had received PCV 7 and /or PCV13. Thirty-nine $(35 \%)$ patients had underlying disease or condition such as malignancy $(n=7)$, post liver transplantation $(n=3)$, congenital heart diseases $(n=3)$, asplenia $(n=2)$ and cerebrospinal fluid leak $(n=1)$. There were 52 penicillin susceptible $S$. pneumoniae isolates, 22 penicillin intermediate isolates and 36 penicillin resistant isolates. The focus of infection was pneumonia $(n=19)$, otitis media $(n=7)$, sinusitis $(n=6)$ and meningitis $(n=4)$, but the majority had no apparent focus and 
Table 2 Clinical characteristics of the patients with Moraxella catarrhalis bacteremia

\begin{tabular}{|c|c|c|c|c|c|c|c|c|c|c|c|c|c|}
\hline & Gender & $\begin{array}{l}\text { age } \\
\text { (month) }\end{array}$ & $\begin{array}{l}\text { BW } \\
(\mathrm{kg})\end{array}$ & Underlying diseases & IS & Devices & $\begin{array}{l}\text { Sick } \\
\text { contact }\end{array}$ & Symptoms & $\begin{array}{l}\text { Pre-existing } \\
\text { antibiotics }\end{array}$ & $\begin{array}{l}\text { Focus of } \\
\text { infection }\end{array}$ & Setting & $\begin{array}{l}\text { ICU } \\
\text { admission }\end{array}$ & Others \\
\hline 1 & female & 5 & 8.3 & Biliary atresia & - & YES (ED tube) & NO & Rhinorrhea Cough & None & Bronchitis & Hospital & NO & - \\
\hline 2 & female & 7 & 3.1 & ELBWI, CLD Multiple malformation & - & NO & YES & Cough & None & Pneumonia & Hospital & YES & Pneumothorax \\
\hline 3 & female & 8 & 9.0 & Bilateral hydronephrosis & - & NO & YES & Vomiting, Diarrhea & None & Unknown & Community & NO & Rotavirus $(+)^{a}$ \\
\hline 4 & male & 9 & 7.7 & none & - & $\begin{array}{l}\text { YES (ETT, NG } \\
\text { tube) }\end{array}$ & NO & Cough & None & Bronchitis & Community & NO & $\operatorname{RSV}(+)^{\mathrm{b}}$ \\
\hline 5 & female & 15 & 10.0 & Glycogen storage disease type $1 b$ & $\begin{array}{l}\text { TAC } \\
\text { mPSL }\end{array}$ & $\begin{array}{l}\text { YES (ETT, NG, } \\
\text { CVC A line) }\end{array}$ & NO & - & None & Pneumonia & Hospital & YES & - \\
\hline 6 & female & 18 & 6.7 & $\begin{array}{l}\text { VACTER association, ELBWI, CLD, } \\
\text { CAVB, DORV/PS/MS }\end{array}$ & - & $\begin{array}{l}\text { YES (ED tube, } \\
\text { PM) }\end{array}$ & NO & Rhinorrhea Cough & None & Pneumonia & Hospital & NO & \\
\hline 7 & male & 20 & 8.8 & $\mathrm{SDH}$, vascular ring & - & YES (NG tube) & NO & $\begin{array}{l}\text { Vomiting, Wheeze } \\
\text { Convulsion }\end{array}$ & None & Bronchitis & Hospital & NO & - \\
\hline 8 & female & 44 & 12.9 & $\begin{array}{l}\text { Congenital intestinal motor } \\
\text { dysfuntion }\end{array}$ & - & YES (CVC) & NO & Cough & None & Pneumonia & Hospital & NO & - \\
\hline
\end{tabular}

indicates concomitant infection with rotavirus diagnosed by rapid antigen test

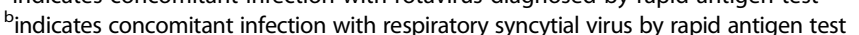

Abbreviations: $B W$ body weight, ELBWI extremely low birth weight infant, CLD chronic lung disease, VACTER Vertebral-Anal-Cardiac-Tracheoesophageal fistula-Renal, CAVB complete atrioventricular block, DORV double outlet right ventricle, PS pulmonary stenosis, MS mitral stenosis, SDH subdural hemorrhage, TAC tacrolimus, mPSL methyl prednisolone, ED elemental diet, ETT endotracheal tube, NG nasogastolic, PM pacemaker, CVC central venous catheter, $A$ line arterial line, $I C U$ intensive care unit, $R S V$ respiratory syncytial virus 
was classified as occult bacteremia $(n=40)$. Of the 84 hospitalized patients (76 \%), nine $(8 \%)$ were admitted to PICU. Twenty-one patients $(24 \%)$ were treated at the outpatient clinic. Empiric antimicrobial therapy is detailed in Additional file 1: Table S1. Of all patients with S. pneumoniae, two patients had recurrences; one was previously immunocompetent with congenital cytomegalovirus infection who developed four episodes of bacteremia, and the other was immunocompromised with medulloblastoma who received surgical, chemoradiotherapy and peripheral blood stem cell transplantation and had a recurrence of bacteremia two weeks after a treatment with piperacillin $(100 \mathrm{mg} / \mathrm{kg} /$ day $)$ for 6 days.

\section{H. influenzae}

There were 22 cases with bacteremia due to $H$. influen$z a e$. All but one was caused by $H$. influenzae type b. Mean age was 25 months and 16 (73\%) were male. Of all the patients included with bacteremia due to $H$. influenzae, only one (5\%) had received the Hib vaccine. Nine (41\%) patients had underlying disease or condition such as trisomy $21(n=2)$, asplenia $(n=1)$, Cornelia-de-Lange syndrome $(n=1)$, Prader-Willi syndrome $(n=1)$, asthma $(n=1)$, leukemoid reaction with unknown cause $(n=1)$, anterior urethral valve with vesicoureteral reflux (Grade III) and neurogenic bladder $(n=1)$, as well as swallowing dysfunction and rhinopharynx reflux with upper airway stricture $(n=1)$. No patient received immunosuppressants or had medical device. Bacteremia was due to nontypeable $H$. influenzae in one case and the rest were due to Hib. Common sites of infection were the central nervous system (meningitis $[n=8]$ ) and lungs (pneumonia $[n=5])$. Of the $14(64 \%)$ patients hospitalized, one patient with meningitis was admitted to PICU. Empiric antimicirobial therapy is detailed in Additional file 1: Table S1. The rate of initiating combination antibiotic therapy was highest (27\%) among three groups.

\section{Comparison of patients' characteristics}

\section{1) M. catarrhalis versus S. pneumoniae}

The results of univariate analysis comparing the characteristics of patients with bacteremia due to $M$. catarrhalis (Moraxella group) and those with bacteremia due to $S$. pneumoniae (Pneumococcus group) are shown in Table 1. Moraxella group had lower body weight, higher rate of patients with underlying diseases or conditions and medical devices, particularly trans-nasal devices, compared to the pneumococcus group $(P=0.016, P=0.005$, $P=0.001$, and $P<0.001$, respectively). In addition, a higher rate of disease onset during hospitalization and lower white blood cell count were noted in the
Moraxella group compared with the pneumococcus group $(P<0.001, P=0.011$, respectively). A higher rate of patients was on antibiotics in the pneumococcus group $(P=0.049)$. The percentage of patients with a history of contact with sick people and symptoms at the time of onset of illness were not different between the two groups $(P=0.472, P=0.281$ [for rhinorrhea], $P=0.274$ [for cough], $P=0.445$ [for convulsion], $P=0.296$ [for vomiting], $P=0.350$ [for diarrhea], respectively). Presence or absence of immunodeficiency and immunosuppressant use was not significantly different between the two groups. Duration of fever after appropriate treatment was approximately one day longer in the Moraxella group than in the pneumococcus group $(P=0.012)$.

2) $M$. catarrhalis versus $H$. influenzae

The results of univariate analyses regarding the relationship between the patients with bacteremia due to $M$. catarrhalis (Moraxella group) and those with bacteremia due to $H$. influenzae (Haemophilus group) are shown in Table 1 . The Moraxella group had a higher rate of patients with disease or condition and medical devices, especially trans-nasal devices, compared with the Haemophilus group $(P=0.039, P<0.001$, and $P<0.001$, respectively). In addition, a lower percentage of the patients were on antibiotics prior to presentation and a higher percentage of those with history of intubation were noted in the Moraxella group compared with the Haemophilus group $(P=0.029, P=0.032$, respectively).

\section{Discussion}

Our study demonstrated that patients with bacteremia due to $M$. catarrhalis were more likely to have underlying diseases or conditions, which necessitated the use of medical devices, such as trans-nasal devices, in comparison with those with bacteremia due to S. pneumoniae or $H$. influenzae. To the best of our knowledge, this is the first report that describes the differences in background and clinical characteristics of $M$. catarralis bacteremia compared with pneumococcal and $H$. influenzae bacteremia.

$M$. catarrhalis is regarded as one of the most common bacterial causes of respiratory tract infections, following $S$. pneumoniae and $H$. influenzae. According to reports from 1992 to 2006, $15-20 \%$ of acute otitis media episodes were caused by $M$. catarrhalis on the basis of cultures of middle ear fluid obtained by tympanocentesis [7, 16-18]. Colonization rate of $M$. catarrhalis in Japanese children attending day-care centers was $34.6 \%$, which followed S. pneumoniae (60.3\%) and H. influenzae $(53.2 \%)$ in the pre-vaccine era [19]. Several reports from countries where PCVs are widely used demonstrated that 
colonization by vaccine serotypes of pneumococcus were decreasing, while colonization by the pneumococcus with non-PCV serotypes $H$. influenzae and $M$. catarrhalis were increasing, resulting in a change in the pathogens that cause otitis media $[7,20,21]$ and sinusitis [7, 22, 23]. However, with the recent inclusion of Hib vaccine and PCV [13] in the national immunization program in Japan, the colonization rate of $M$. catarrhalis may increase in the near future.

M. catarrhalis is an important pathogen in respiratory tract infections in children, but bacteremia is rare [24]. Several recent reports revealed that previously healthy immunocompetent patients developed Moraxella bacteremia at a constant rate [24-27]. In the current study, however, only one previously healthy child developed bacteremia due to $M$. catarrhalis and the remaining seven patients had underlying disease. A previous report described 11 cases of bacteremia due to $M$. catarrhalis and noted that it should be considered in febrile children with underlying immunodeficiency and upper respiratory tract infection [8]. Ioannidis et al. noted that nearly $30 \%$ of patients with $M$. catarrhalis bacteremia had underlying respiratory factors including tracheostomy or dependence on mechanical ventilation, and acute respiratory infections such as viral pneumonia [28]. The authors also described an association with chronic neurological debilities as one of the predisposing factors [28]. Similarly, we found that a quarter of the patients had underlying respiratory diseases, and three out of eight patients had chronic neurological problems. More specifically, our study highlights the association of medical devices, particularly trans-nasal devices, as independent factors associated with bacteremia due to M. catarrhalis compared with S. pneumoniae or $H$. influenzae. Most isolates of $M$. catarrhalis produce beta-lactamases in contrast to S. pneumoniae which acquire modest ampicillin resistance through modification of the penicillin binding proteins. Our findings suggests that modification of empiric coverage to cover penicillinase producing $M$. catarrhalis is a consideration for patients with underlying disorders with trans-nasal devices who develop respiratory tract infections.

The pathogenic association of medical devices and invasive disease caused by $M$. catarrhalis is unclear. The nasopharyngeal colonization of $M$. catarrhalis is quite common among children, especially through infancy, and $M$. catarrhalis forms biofilms and has been identified in the middle ears of children with otitis media [7]. Adherence of $M$. catarrhalis to the respiratory epithelium and mucin molecules of the nasopharynx and the middle ear is known to be a key step in the initiation of infection $[29,30]$. M. catarrhalis has also been found intracellularly in subepithelial cell and lymphoid tissue [31], with a possibility that the breach in the integrity of the mucosal barrier by medical devices may also contribute to the development of invasive infections. A previous report identified the presence of endotracheal tubes and frequent endotracheal tube suctioning as risk factors for developing nosocomial infection due to $M$. catarrhalis, which support our findings [32]. Moreover, another report describes the association of respiratory tract fragility with bacteremic pneumonia caused by $M$. catarrhalis may reflect increased rates of oropharyngeal colonization, enhancement of bacterial adherence to abnormal epithelium, and increased susceptibility of pulmonary parenchyma to infection [28, 33]. According to a study using an animal model, type IV pili of $M$. catarrhalis plays a crucial role in colonization of the nasopharyngeal mucosa and biofilm formation [34]. In addition, the biofilms produced by $M$. catarrhalis are similar to the complicated structures produced by several bacteria, such as Pseudomonas aeruginosa. Biofilm formation is important in considering infections associated with foreign body, such as endotracheal tube [34, 35]. However, it has yet to be revealed whether biofilms contribute to pathogenesis of infections caused by $M$. cararrhalis. Further basic and clinical studies are needed to clarify our hypothesis.

A previous report that described the clinical and laboratory aspects of $M$. catarrhalis bacteremia in 17 children showed that most patients with $M$. catarrhalis bacteremia had modest elevation in body temperature (mean \pm SD: $37.3 \pm 1.2^{\circ} \mathrm{C}$ ) and leukocytosis (mean \pm SD: $12,070 \pm 7569 / \mu \mathrm{L}$ ) [24]. In the current study, although all patients had a maximum temperature $>38.0{ }^{\circ} \mathrm{C}$, the fever tended to be mild and leukocyte count was 14,774 $\pm 13,750 / \mu \mathrm{L}$ (mean $\pm \mathrm{SD}$ ), denoting similar tendency with the previous report. Such data suggests that M. catarrhalis is associated with relatively mild inflammation. However, the patients' immune statuses including neutrophil function, complement and humoral immunity, which may influence the degree of inflammation, were not fully investigated in the current study.

We acknowledge that there are several limitations to this study. First, this study is a retrospective case-control-control study based on a single center experience in Japan. In addition, there were few patients with $M$. catarrhalis bacteremia. Thus, we performed only univariate analyses, and therefore our results should be interpreted carefully. Second, this study was conducted on patients with bacteremia due to $H$. influenzae and S. pneumoniae during a transition period of the national immunization program in Japan before and after introduction of $\mathrm{Hib}$ vaccine and $\mathrm{PCV}$, which may influence patient background. Finally, the study evaluated the clinical characteristics of the Moraxella bacteremia patients against those with pneumococcal or Haemophilus bacteremia. Therefore, our study is likely to underestimate the risk factors that are common to the development of 
bacteremia caused by all three organisms. Hereafter, collection and evaluation of national surveillance data regarding Moraxella bacteremia patients are required to clarify epidemiological changes in the post-vaccine era and the risk factors of patients with Moraxella bacteremia.

\section{Conclusions}

Patients with bacteremia due to $M$. catarrhalis were likely to have underlying diseases or conditions requiring medical devices, particularly trans-nasal devices.

\section{Additional file}

Additional file 1: Table S1. Empiric antimicrobial therapy among three groups. (DOCX 35 kb)

\begin{abstract}
Abbreviations
Hib: H. influenzae type b; IHD: Invasive haemophilus disease; IPD: Invasive pneumococcal diseases; NCCHD: National Center for Child Health and Development; PCV: Pneumococcal conjugate vaccine; PICU: Pediatric intensive care unit.; SD: Standard deviation.
\end{abstract}

\section{Competing interests}

The authors declare that they have no competing interests.

\section{Authors' contributions}

TF contributed conception and design of the study, data collection, interpretation of data for this study, and drafting of this manuscript. El contributed design of the study and statistical analyses. IM contributed interpretation of data for this study, and draft of this manuscript and manuscript revision. All authors approved of the final manuscript.

\section{Acknowledgements}

We would like to thank Dr. Julian Tang of the Department of Education for Clinical Research, National Center for Child Health and Development, for proofreading and editing this manuscript.

\section{Funding source}

This manuscript was supported by funding from the National Center for Child Health and Development to IM (24-11-3) and TF (24-31).

\section{Author details}

'Division of Infectious Diseases, Department of Medical Subspecialties, National Center for Child Health and Development, 2-10-1 Okura, Setagayaku, Tokyo 1578535, Japan. ${ }^{2}$ Center for Clinical Research and Development, National Center for Child Health and Development, 2-10-1 okura, Setagayaku, Tokyo 1578535, Japan.

Received: 22 August 2015 Accepted: 2 February 2016 Published online: 09 February 2016

\section{References}

1. Centers for Disease Control and Prevention (CDC). Invasive pneumococcal disease in children 5 years after conjugate vaccine introduction-eight states, 1998-2005. MMWR Morb Mortal Wkly Rep. 2008;57(6):144-8.

2. Centers for Disease Control and Prevention (CDC). Invasive pneumococcal disease in young children before licensure of 13-valent pneumococcal conjugate vaccine - United States, 2007. MMWR Morb Mortal Wkly Rep. 2010;59(9):253-7.

3. Moore MR, Link-Gelles R, Schaffner W, Lynfield R, Lexau C, Bennett NM, et al. Effect of use of 13-valent pneumococcal conjugate vaccine in children on invasive pneumococcal disease in children and adults in the USA: analysis of multisite, population-based surveillance. Lancet Infect Dis. 2015;15(3):301-9.

4. Centers for Disease Control and Prevention (CDC). Progress toward elimination of Haemophilus influenzae type b invasive disease among infants and children-United States, 1998-2000. MMWR Morb Mortal Wkly Rep. 2002;51(11):234-7.

5. Watt JP, Wolfson LJ, O'Brien KL, Henkle E, Deloria-Knoll M, McCall N, et al. Burden of disease caused by Haemophilus influenzae type $b$ in children younger than 5 years: global estimates. Lancet. 2009;374(9693):903-11.

6. Institute of Infectious Diseases and Tuberculosis and Infectious Disease Control Division. Invasive Haemophilus Influenzae and Streptococcus pneumoniae infections, as of August 2014. In: Ministry of Health Labour and Welfare, vol. 35. 2014. http://www0.nih.go.jp/niid/idsc/iasr/35/416j.pdf. Accessed 8 Feb 2016.

7. Murphy TF, Parameswaran GI. Moraxella catarrhalis, a human respiratory tract pathogen. Clin Infect Dis. 2009;49(1):124-31.

8. Abuhammour WM, Abdel-Haq NM, Asmar BI, Dajani AS. Moraxella catarrhalis bacteremia: a 10-year experience. South Med J. 1999;92(11):1071-4.

9. Baraff $L J$, Oslund S, Prather M. Effect of antibiotic therapy and etiologic microorganism on the risk of bacterial meningitis in children with occult bacteremia. Pediatrics. 1993:92(1):140-3.

10. American Academy of Pediatrics. Immunization in special clinical circumstances. In: Pickering LK, Baker CJ, Kimberlin DW, Long SS, editors. Red Book. 29th ed. Elk Grove Village, IL: American Academy of Pediatrics; 2012. p. 69-109.

11. CLSI. Performance Standards for Antimicrobial Susceptibility Testing M100S19; Nineteenth Informational Supplement, vol. 29. Wayne: Clinical and Laboratory Standards Institute; 2009.

12. Saitoh A, Okabe N. Current issues with the immunization program in Japan: can we fill the "vaccine gap"? Vaccine. 2012;30(32):4752-6.

13. Saitoh A, Okabe N. Recent progress and concerns regarding the Japanese immunization program: addressing the "vaccine gap". vaccine. 2014;32(34):4253-8.

14. Japan Pediatric Society. Vaccination Schedule Recommended by the Japan Pediatric Society. In: Japan Pediatric Society web site. Japan Pediatric Society. 2015 http://www.jpeds.or.jp/uploads/files/JPS\%20Vaccine\% 20Schedule\%20English\%2020140317.pdf. Accessed 8 Feb 2016.

15. R Development Core Team. R: A Language and Environment for Statistical Computing. Vienna, Austria: the R Foundation for statistical Computing; 2013. Available online at http://www.R-project.org/.

16. Del Beccaro MA, Mendelman PM, Inglis AF, et al. Bacteriology of acute otitis media: a new perspective. J Pediatr Jan. 1992;120(1):81-4

17. Kilpi T, Herva E, Kaijalainen T, Syrjanen R, Takala AK. Bacteriology of acute otitis media in a cohort of Finnish children followed for the first two years of life. Pediatr Infect Dis J. 2001;20(7):654-62.

18. Ruohola A, Meurman O, Nikkari S, Skottman T, Salmi A, Waris M, et al. Microbiology of acute otitis media in children with tympanostomy tubes: prevalences of bacteria and viruses. Clin Infect Dis. 2006;43(11):1417-22.

19. Masuda K, Masuda R, Nishi J, Tokuda K, Yoshinaga M, Miyata K Incidences of nasopharyngeal colonization of respiratory bacterial pathogens in Japanese children attending day-care centers. Pediatr Int. 2002;44(4):376-80

20. Block SL, Hedrick J, Harrison CJ, Tyler R, Smith A, Findlay R, et al. Community-wide vaccination with the heptavalent pneumococcal conjugate significantly alters the microbiology of acute otitis media. Pediat Infect Dis J. 2004;23(9):829-33.

21. Casey JR, Pichichero ME. Changes in frequency and pathogens causing acute otitis media in 1995-2003. Pediatr Infect Dis J. 2004;23(9):824-8.

22. Brook I, Foote PA, Hausfeld JN. Frequency of recovery of pathogens causing acute maxillary sinusitis in adults before and after introduction of vaccination of children with the 7-valent pneumococcal vaccine. J Med Microbiol. 2006;55(Pt 7):943-6.

23. Brook I, Gober AE. Frequency of recovery of pathogens from the nasopharynx of children with acute maxillary sinusitis before and after the introduction of vaccination with the 7 -valent pneumococcal vaccine. Int $J$ Pediatr Otorhinolaryngol. 2007;71(4):575-9.

24. Ahmed A, Broides A, Givon-Lavi N, Peled N, Dagan R, Greenberg D. Clinical and laboratory aspects of Moraxella catarrhalis bacteremia in children. Pediatr Infect Dis J. 2008;27(5):459-61

25. McGregor K, Chang BJ, Mee BJ, Riley TV. Moraxella catarrhalis: clinical significance, antimicrobial susceptibility and BRO beta-lactamases. Eur J Clin Microbiol Infect Dis. 1998:17(4):219-34.

26. Meyer GA, Shope TR, Waecker Jr NJ, Lanningham FH. Moraxella (Branhamella) catarrhalis bacteremia in children. A report of two patients and review of the literature. Clin Pediatr. 1995;34(3):146-50. 
27. Enright MC, McKenzie H. Moraxella (Branhamella) catarrhalis-clinical and molecular aspects of a rediscovered pathogen. J Med Microbiol. 1997:46(5):360-71.

28. Ioannidis JP, Worthington M, Griffiths JK, Snydman DR. Spectrum and significance of bacteremia due to Moraxella catarrhalis. Clin Infect Dis. 1995:21(2):390-7.

29. Verduin CM, Hol C, Fleer A, van Dijk H, van Belkum A. Moraxella catarrhalis: from emerging to established pathogen. Clin Microbiol Rev. 2002;15(1):125-44.

30. Bernstein JM, Reddy M. Bacteria-mucin interaction in the upper aerodigestive tract shows striking heterogeneity: implications in otitis media, rhinosinusitis, and pneumonia. Otolaryngol Head Neck Surg Apr. 2000;122(4):514-20.

31. Heiniger N, Spaniol V, Troller R, Vischer M, Aebi C. A Reservoir of Moraxella catarrhalis in Human Pharyngeal Lymphoid Tissue. J Infect Dis. 2007;196(7): 1080-7.

32. Cook PP, Hecht DW, Snydman DR. Nosocomial Branhamella catarrhalis in a paediatric intensive care unit: risk factors for disease. J Hosp Infect. 1989. 13(3):299-307.

33. Hager $\mathrm{H}$, Verghese A, Alvarez S, Berk SL. Branhamella catarrhalis respiratory infections. Rev Infect Dis. 1987;9(6):1140-9.

34. Luke NR, Jurcisek JA, Bakaletz LO, Campagnari AA. Contribution of Moraxella catarrhalis type IV pili to nasopharyngeal colonization and biofilm formation. Infect Immun. 2007;75(12):5559-64.

35. Davey ME, O'Toole GA. Microbial biofilms: from ecology to molecular genetics. Microbiol Mol Biol Rev. 2000;64(4):847-67.

\section{Submit your next manuscript to BioMed Central and we will help you at every step:}

- We accept pre-submission inquiries

- Our selector tool helps you to find the most relevant journal

- We provide round the clock customer support

- Convenient online submission

- Thorough peer review

- Inclusion in PubMed and all major indexing services

- Maximum visibility for your research

Submit your manuscript at www.biomedcentral.com/submit 